\title{
UCRL-PROC-216219
}

LAWRENCE LIVERMORE N A T IO N A L LABORATORY

\section{Experimental Benchmarking of Pu Electronic Structure}

J. G. Tobin, K. T. Moore, B. W. Chung, M. A. Wall, A. J. Schwartz, B. B. Ebbinghaus, M. T. Butterfield, N. E. Teslich, Jr., R. A. Bliss, S. A. Morton, S. W. Yu, T. Komesu, G. D. Waddill, G. van der Laan, A. L. Kutepov

October 17, 2005

2005 Fall MRS Meeting

Boston, MA, United States

November 28, 2005 through December 2, 2005 
This document was prepared as an account of work sponsored by an agency of the United States Government. Neither the United States Government nor the University of California nor any of their employees, makes any warranty, express or implied, or assumes any legal liability or responsibility for the accuracy, completeness, or usefulness of any information, apparatus, product, or process disclosed, or represents that its use would not infringe privately owned rights. Reference herein to any specific commercial product, process, or service by trade name, trademark, manufacturer, or otherwise, does not necessarily constitute or imply its endorsement, recommendation, or favoring by the United States Government or the University of California. The views and opinions of authors expressed herein do not necessarily state or reflect those of the United States Government or the University of California, and shall not be used for advertising or product endorsement purposes. 


\title{
Experimental Benchmarking of Pu Electronic Structure
}

\author{
J.G. Tobin ${ }^{1, *}$, K.T. Moore ${ }^{1}$, B.W. Chung ${ }^{1}$, M.A. Wall ${ }^{1}$, A.J. Schwartz ${ }^{1}$, B.B. Ebbinghaus ${ }^{1}$, \\ M.T. Butterfield ${ }^{1}$, N.E. Teslich Jr. ${ }^{1}$, R.A. Bliss ${ }^{1}$, S.A. Morton ${ }^{1, \#}$, S.W. Yu ${ }^{1}$, \\ T. Komesu' ${ }^{2}$, G.D. Waddill' ${ }^{2}$ G. van der Laan $^{3}$, and A.L. Kutepov ${ }^{4}$ \\ 1. Lawrence Livermore National Laboratory, Livermore, CA, USA 94550 \\ 2. University of Missouri-Rolla, Rolla, MO \\ 3. Synchrotron Radiation Source, Daresbury Laboratory, Warrington, WA4 4AD, UK \\ 4. Russian Federation Nuclear Center, Inst. of Tech. Physics (VNIITF), Snezhinsk, Russia \\ *Contact author: Tobin1@,LLNL.Gov \\ \#Present Address: Advanced Light Source, LBNL, Berkeley, CA
}

\begin{abstract}
The standard method to determine the band structure of a condensed phase material is to (1) obtain a single crystal with a well defined surface and (2) map the bands with angle resolved photoelectron spectroscopy (occupied or valence bands) and inverse photoelectron spectroscopy (unoccupied or conduction bands). Unfortunately, in the case of $\mathrm{Pu}$, the single crystals of $\mathrm{Pu}$ are either nonexistent, very small and/or having poorly defined surfaces. Furthermore, effects such as electron correlation and a large spin-orbit splitting in the $5 \mathrm{f}$ states have further complicated the situation. Thus, we have embarked upon the utilization of unorthodox electron spectroscopies, to circumvent the problems caused by the absence of large single crystals of $\mathrm{Pu}$ with well-defined surfaces. Our approach includes the techniques of resonant photoelectron spectroscopy [1], x-ray absorption spectroscopy $[1,2,3,4]$, electron energy loss spectroscopy $[2,3,4]$, Fano Effect measurements [5], and Bremstrahlung Isochromat Spectroscopy [6], including the utilization of micro-focused beams to probe single-crystallite regions of polycrystalline Pu samples. $[2,3,6]$
\end{abstract}

\section{Motivation}

The objective of this work is to develop and apply advanced diagnostics to the understanding of aging of Pu. Advanced characterization techniques such as photoelectron and $\mathrm{x}$-ray absorption spectroscopy will provide fundamental data on the electronic structure of $\mathrm{Pu}$ phases. These data are crucial for the validation of the electronic structure methods. The fundamental goal of this project is to narrow the parameter space for the theoretical modeling of Pu aging. The short-term goal is to perform experiments to validate electronic structure calculations of $\mathrm{Pu}$. The long-term goal is to determine the effects of aging upon the electronic structure of $\mathrm{Pu}$.

Many of the input parameters for aging models are not directly measurable. These parameters will need to be calculated or estimated. Thus a First Principles-Approach Theory is needed, but it is unclear what terms are important in the Hamiltonian. ( $\mathrm{H} \square=$ E $\square$ ) Therefore, experimental data concerning the $5 f$ electronic structure are needed, to determine which terms in the Hamiltonian are important. 


\section{Present Status}

IIa. Present Status: Spectroscopic Observation of Aging in Pu

Resonant Photoemission, a variant of Photoelectron Spectroscopy, has been demonstrated to have sensitivity to aging of Pu samples. [1] The spectroscopic results have been correlated with resistivity measurements and shown to be the fingerprint of mesoscopic or nanoscale internal damage in the Pu physical structure. This means that a spectroscopic signature of internal damage due to aging in $\mathrm{Pu}$ has been established. The details of this study are provided elsewhere, including another chapter in this proceedings. [7]

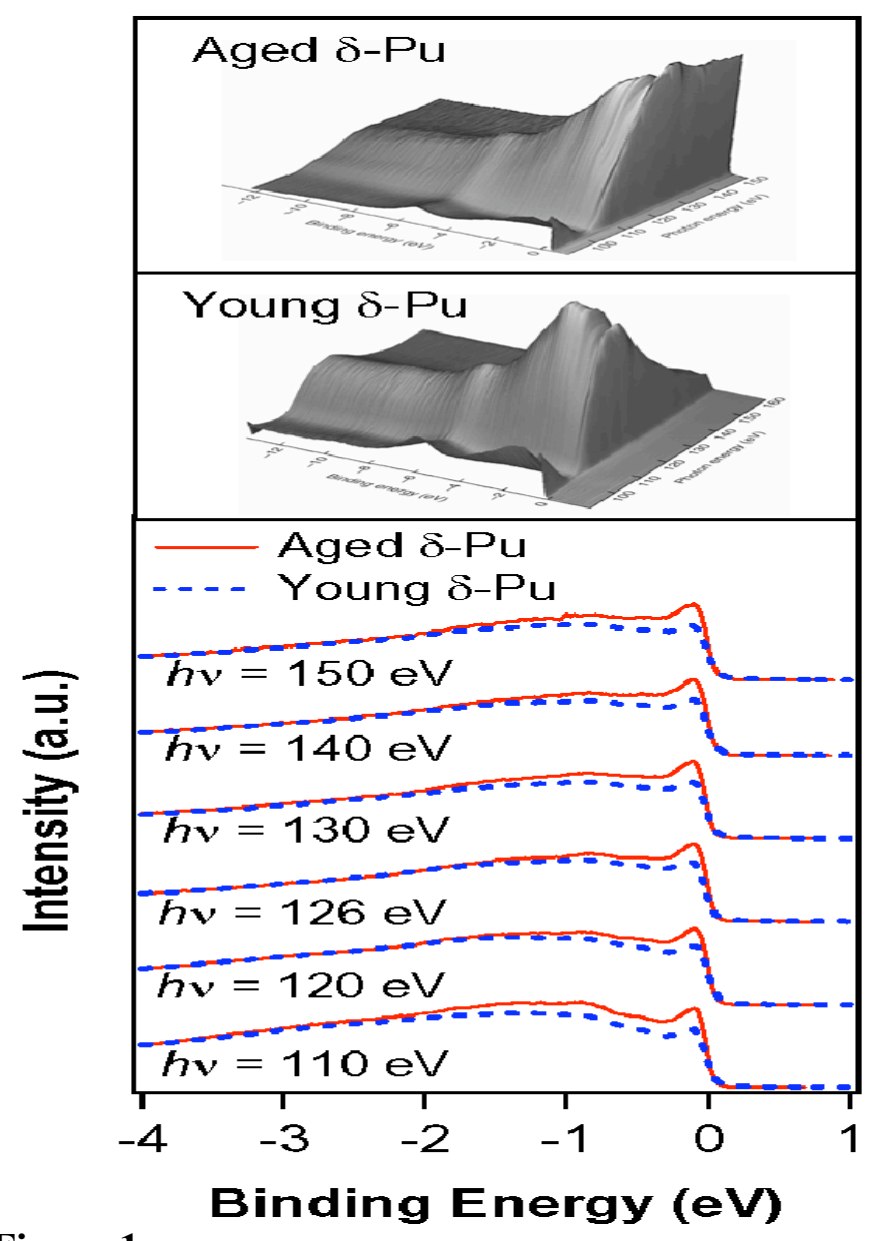

\section{Figure 1}

The RESPES results for a young, highly purified $\square \mathrm{Pu}(\mathrm{Ga})$ sample are compared with the corresponding measurements of an aged $\square-\mathrm{Pu}(\mathrm{Ga})$, that is approximately 10 years old. In the topmost panels are the pseudo-three dimensional plots, with binding energy ( 0 to -12 $\mathrm{eV}$ ) and photon energy (100 to 150 or $160 \mathrm{eV}$ ) as the in plane axes and the out of plane axis being intensity. Here, normalization is via flux measurements using a gold grid upsteam from the photoemission site. In the lower panel, comparison of the young (blue dashes) and aged (red lines) samples is made at specific photon energies, over the resonance regime photon energy range. In these lower panels, normalization is made at points above the Fermi Energy and at a binding energy of $-4 \mathrm{eV}$. 


\section{IIb. Present Status: Fundamental Studies of Pu Electronic Structure}

Understanding the behavior of $5 \mathrm{f}$ electrons remains an important and unachieved goal of condensed matter physics. Recently, there has been a surge of interest in the actinides, particularly plutonium, driven by the variety of exotic behaviors exhibited by these systems, prompting both theoretical and experimental investigations of $5 f$ metals and compounds. Intriguingly, investigations of these exotic behaviors, such as the profoundly large increase in atomic volume that occurs at plutonium or the discovery of $\mathrm{Pu}$-based superconductivity, or observation of previously unmeasured quantities such as the phonon dispersion in $\mathrm{Pu}$, all point back to the $5 \mathrm{f}$ electronic structure as the cause or origin of the anomalies and strange behavior. [Reference 4 and references therein]

We are unraveling the enigma of $\mathrm{Pu}$ electronic structure. Sixty years after its discovery by E.O. Lawrence, the mystery of the electronic structure of $\mathrm{Pu}$ is finally being resolved. In a series of experiments and linked theoretical modeling, the range of possible solutions for $\mathrm{Pu}$ electronic structure has been dramatically reduced.

Our approach is to experimentally determine which potential terms are the largest. (Feynman says: "Know your Hamiltonian!")

$$
H \square=-\left(\square^{2} / 2 m\right) \square+V \square, \quad \text { where } V=V_{1}+V_{2}+V_{3}+V_{4}+\ldots
$$

From the studies described below (Figures 2-4), we have proven the following.

1. $\mathrm{Pu}$ is a jj-skewed Intermediate Coupling case, NOT LS (Russell-Saunders).

2. $\mathrm{n}=5$ for the $\mathrm{Pu} 5 \mathrm{f}$ states.

3. Spin orbit splitting dominates delocalization effects: $\mathrm{V}_{\mathrm{SO}}>\mathrm{V}_{\text {Delocalization }}$

The remaining issues for $\mathrm{Pu}$ electronic structure are mainly those of electron correlation effects. Based upon the success of magnetic methods in explaining the physical properties of the different phases of $\mathrm{Pu}$ [8-10] it is possible to hypothesize that for $\mathrm{F}-\mathrm{Pu}$ there are strong indications that $\mathrm{V}_{\mathrm{MAG}}$ perturbs $\mathrm{V}_{\text {SO }}$ and $\mathrm{V}_{\mathrm{MAG}}>\mathrm{V}_{\text {Delocalization. In }}$ $\mathrm{Pu}$, we expect to observe large but counter aligned spin and orbital polarizations or magnetic moments within the $5 \mathrm{f}$ manifold. The counter alignment should lead to substantial cancellation. However, there would need to be an additional shielding or cancellation going on in $\square-\mathrm{Pu}$, such as Kondo Shielding, Spin Fluctuation, NonCollinearity, or Averaging. Alternatively, there is the possibility that there are no magnetic effects and that the electron correlation is a type of Kondo interaction best described by Dynamical Mean Field Theory. $[11,12]$ We can resolve these last two issues with the Fano Effect and Bremstrahlung Isochromat Spectroscopy measurements, as will be described below. 
Key point \#1: The 5 f spin-orbit splitting is large (Pu has a large negative $<$ w110 $>$.

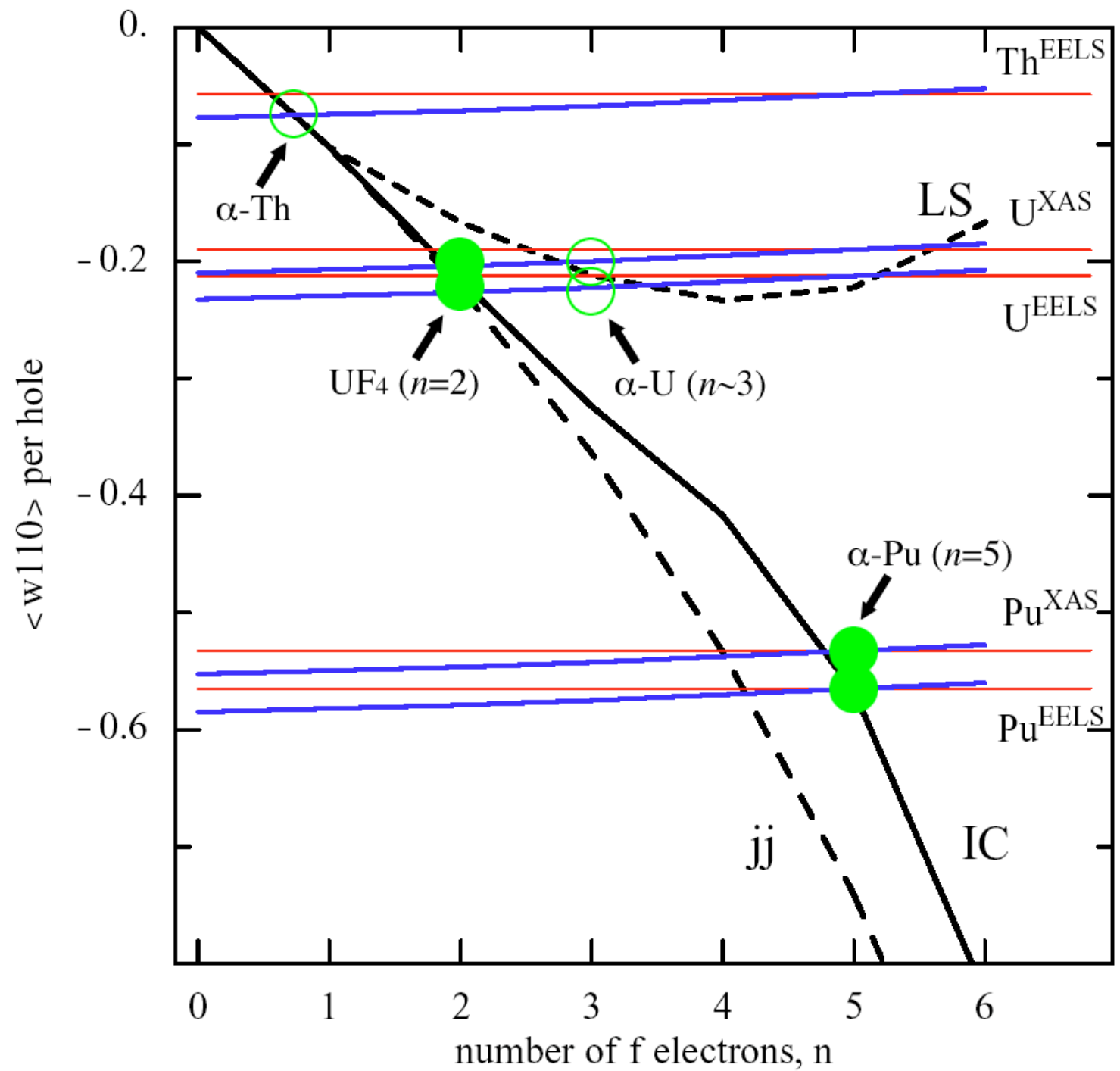

Figure 2

The spin-orbit expectation value $<\mathrm{w} 110>$ as a function of the number of $5 f$ electrons $(n)$, for the $4 \mathrm{~d}$ to $5 \mathrm{f}$ transitions, is shown here. The results of three different theoretical coupling schemes are plotted; $L S$ (short dash), jj (long dash), and intermediate (solid). Experimental results are shown as circles (fixed $\mathrm{n}$ values) or as lines to include the effect of varying $\mathrm{n}$. The red horizontal lines are without the additional correction term $\square$ and the blue lines are including the additional correction term $\square$. See Reference 3 for the details. It is clear: the Pu results are only consistent with a jj-limit or a jj-skewed intermediate case result. There is a large spin-orbit splitting in the $\mathrm{Pu} 5 \mathrm{f}$ states. 
Key point \#2: The number of 5 f electrons is 5

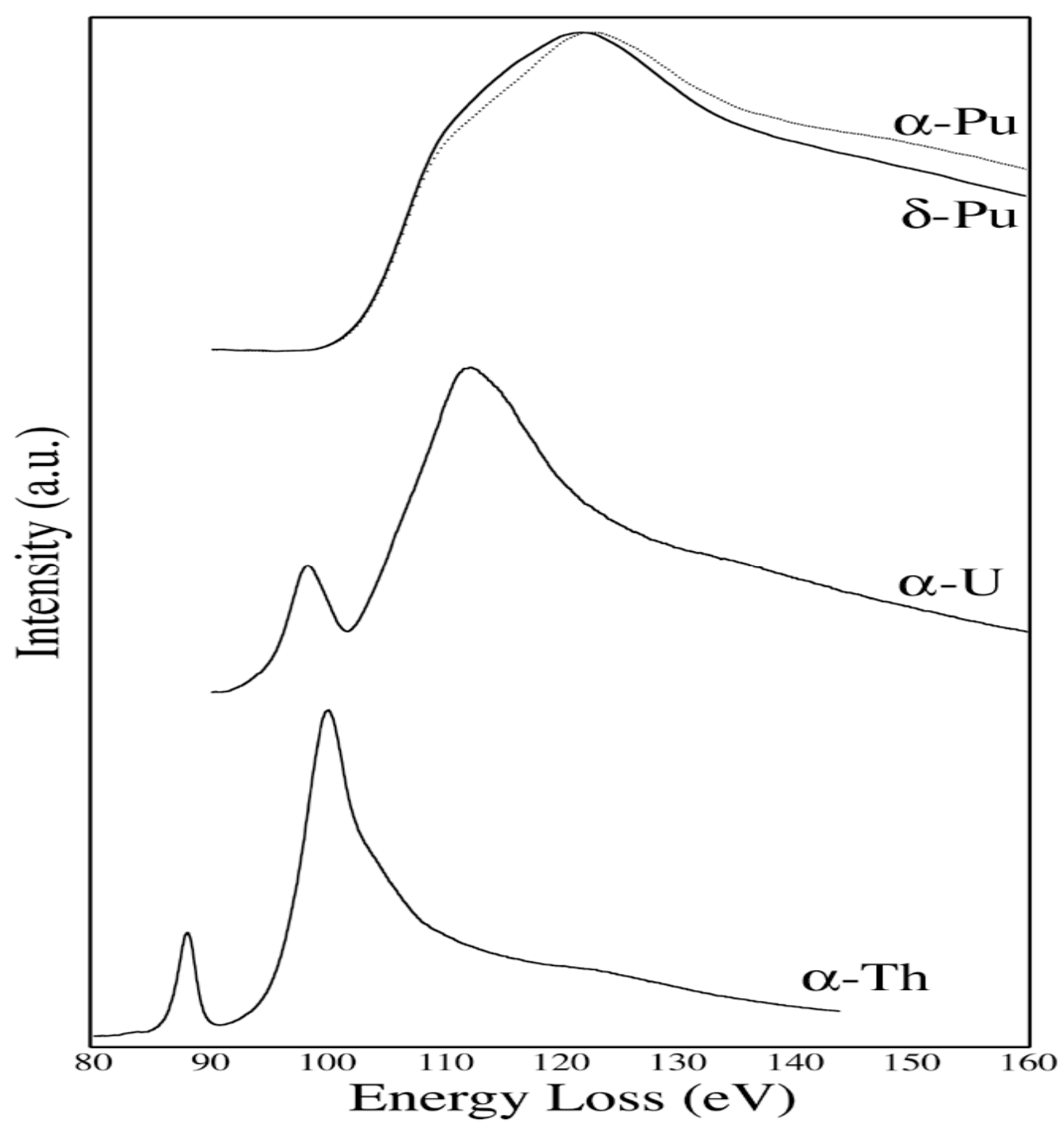

\section{Figure 3}

The $\mathrm{O}_{4,5}(5 \mathrm{~d} \square 5 \mathrm{f}$ ) absorption edges from $\square-\mathrm{Th}, \square-\mathrm{U}, \square-\mathrm{Pu}$ and $\square-\mathrm{Pu}$ acquired by Electron Energy Loss Spectroscopy (EELS) in a Transmission Electron Microscope (TEM). These spectra were collected at an accelerating voltage of $297 \mathrm{keV}$ with an energy resolution of $0.8 \mathrm{eV}$. Note the pre-peak in both $\mathrm{Th}$ and $\mathrm{U}$, consistent with the $4 \mathrm{~d}$ to $4 \mathrm{f}$ transitions in the rare earths. The presence of two edges (below and above $110 \mathrm{eV}$ ) and the absence of the pre-peak in Pu signals that the $5 f$ states in Pu are one electron away from filling a sub-level. In this case, the sublevel is the $5 f^{5 / 2}$ manifold, which can hold up to six electrons. Thus $\mathrm{n}=5$ in Pu. See Reference 2 for details. 
Key Point \#3: $V_{\text {So }}>V_{\text {Delocalization }}$-there are two lobes in the $5 f$ DOS in Pu.

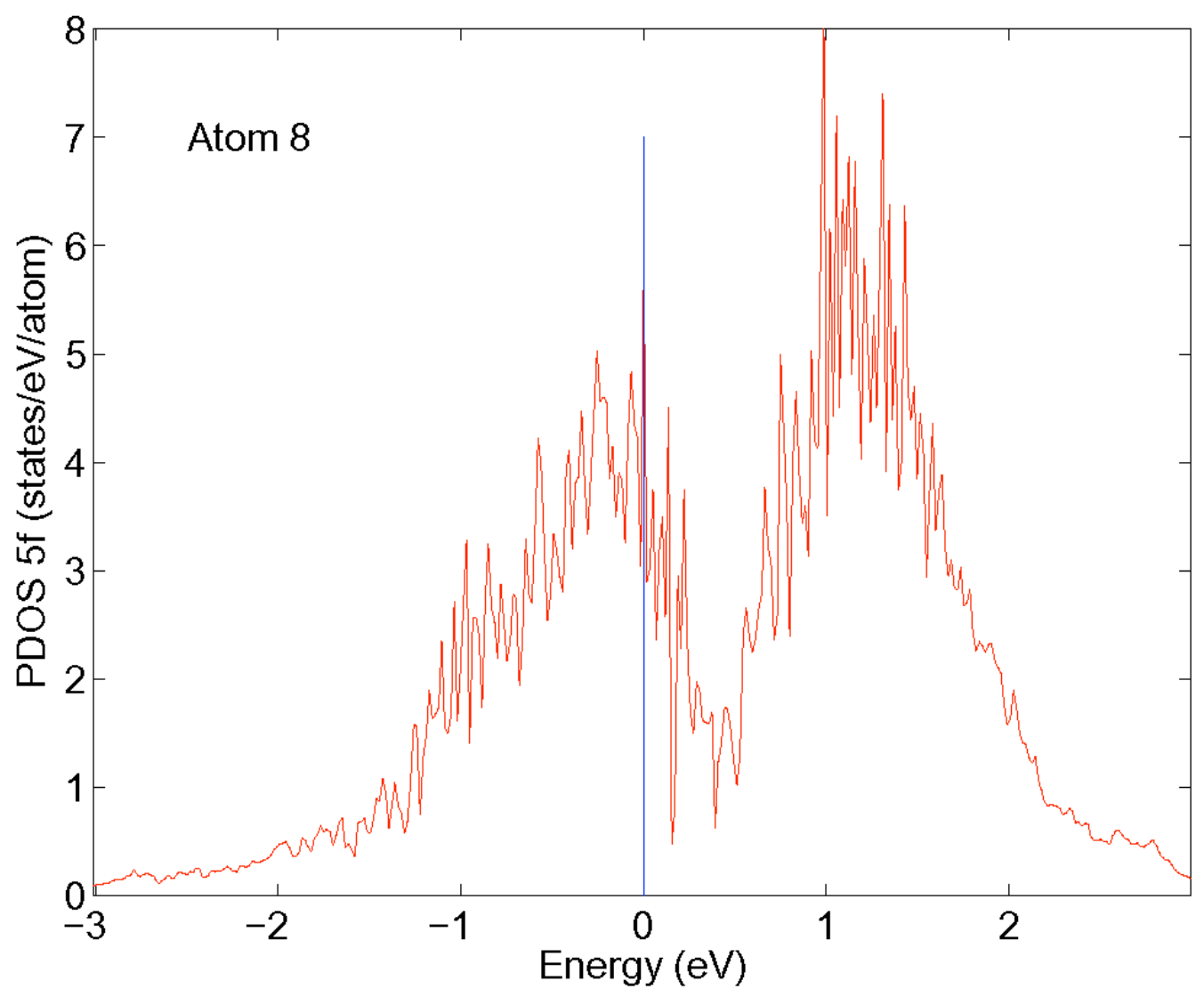

\section{Figure 4}

Non-magnetic calculations of the density of states (DOS) for $\square-\mathrm{Pu}$ are shown here, for atom 8 of the 16 atom unit cell of $\square-\mathrm{Pu}$. These calculations were performed by A.

Kutepov of VNIITF in Russia and included results for all 8 unique sites within the 16 atom unit cell of $\square-\mathrm{Pu}$. States below the Fermi Level (negative energies) are occupied and states above the Fermi Level (positive energies) are unoccupied. The Fermi Level is at 0 $\mathrm{eV}$. The strong retention of the spin orbit splitting indicates that delocalization and hybridization are secondary effects relative to the spin orbit splitting in the $\mathrm{Pu} 5 \mathrm{f}$ states. See Reference 4 for details. 


\section{Future Measurements \\ IIIa. Nano-focused Bremstrahling Isochromat Spectroscopy (nBIS)}

The details of the electronic structure of Pu remain undefined. While chemically toxic and highly radioactive, $\mathrm{Pu}$ may be the most scientifically interesting element in the periodic table. It's properties include the following: six different phases, close to each other in energy and sensitive to variations of temperature, pressure and chemistry; the face-centered-cubic phase (delta) is the least dense; $\mathrm{Pu}$ expands when it solidifies from the melt; and it is clearly the nexus of the actinide binary phase diagram of the actinides. In a sense, it is the boundary between the light (ostensibly delocalized 5f electrons) and heavy (ostensibly localized or correlated $5 \mathrm{f}$ electrons) actinide elements, but this is an over-simplification. The localized atomic $5 \mathrm{f}$ states are naturally correlated, but important regimes of correlated electron states are conceivable as extended states on the delocalized side of the possible Mott transition. The proximity to this crossover may be the driving force behind all these exotic properties. Pu remains of immense technological importance and the advancement to a firm, scientific understanding of the electronic structure of $\mathrm{Pu}$ and its compounds, mixtures, alloys and solutions is a crucial issue. Moreover, while there are a number of ongoing experimental efforts directed at determining the occupied (valence band, below the Fermi Energy) electronic structure of $\mathrm{Pu}$, there is essential no experimental data on the unoccupied (conduction band, above the Fermi Energy) electronic structure of $\mathrm{Pu}$.

We have begun an experimental effort to help resolve the controversy regarding the electronic structure of $\mathrm{Pu}$. [6] The objective of this effort is to determine the conduction band (unoccupied) electronic structure of $\mathrm{Pu}$ and other actinides (and possibly rare earths as well), in a phase specific fashion and emphasizing bulk contributions. Moreover, the conduction band (unoccupied) electronic structure is the missing link in studies of Pu. As illustrated in Figure 5 below, Bremstrahling Isochromat Spectroscopy or BIS is the best way to determine the unoccupied electronic structure of the actinides. While experimental BIS data exists for Th and $\mathrm{U}$, there is no such data for Pu! By using a nano- or micro-focussed beam of electrons as the excitation source (thus nBIS), we will be able to access single-crystalline regions of polycrystalline samples, permitting the determination in a phase specific fashion.

The central technique is BIS, or high energy Inverse Photoelectron Spectroscopy. BIS is the high-energy variant of inverse photoelectron spectroscopy (IPES: electron in, photon out), which is essentially the time reversal of photoelectron spectroscopy (photon in, electron out), as illustrated in Figure 6. IPES can be used to follow the dispersion of electronic states in ordered samples. Owing to its low energies, IPES is usually very surface and band sensitive. However, by working at higher energies, we will sample preferentially for the bulk density of states, downgrading the impact of surface and band effects. Thus, from BIS, we would have a direct measure of the conduction band or unoccupied electronic structure of the bulk Pu.

A more extensive discussion of this experiment will be provided in another chapter of the proceedings volume, by M. Butterfield et al. 

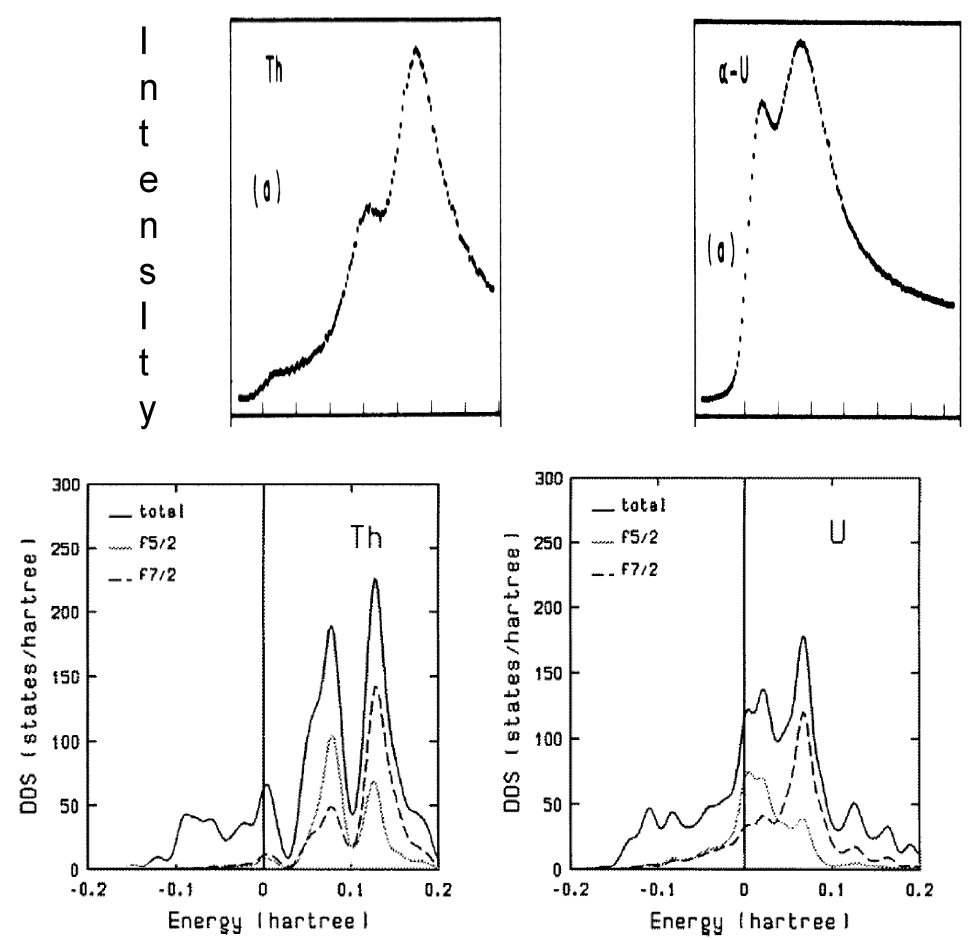

\section{Unoccupied density of states of Th and $U$ from Bremstrahlung Isochromat Spectroscopy (BIS) by Baer and Lang[13].}

Occupied (negative energies) and unoccupied (positive energies) Density of States of Th and $U$ calculated by Penicaud [14].

\section{Figure 5:}

The BIS and calculated electronic structure of Th and U. For each of these elements, there is strong agreement between the experimental and theoretical results, particularly the presence of the spin-orbit split $5 \mathrm{f} 5 / 2$ and $5 \mathrm{f} 7 / 2$ peaks. The detail is greater in the calculations than in the experiment: the BIS experiment is broadened but retains the essence of the spin orbit split peaks. The BIS spectra also exhibit a Fermi cutoff at zero Energy: the transitions cannot go into occupied states. (This will be demonstrated below.)

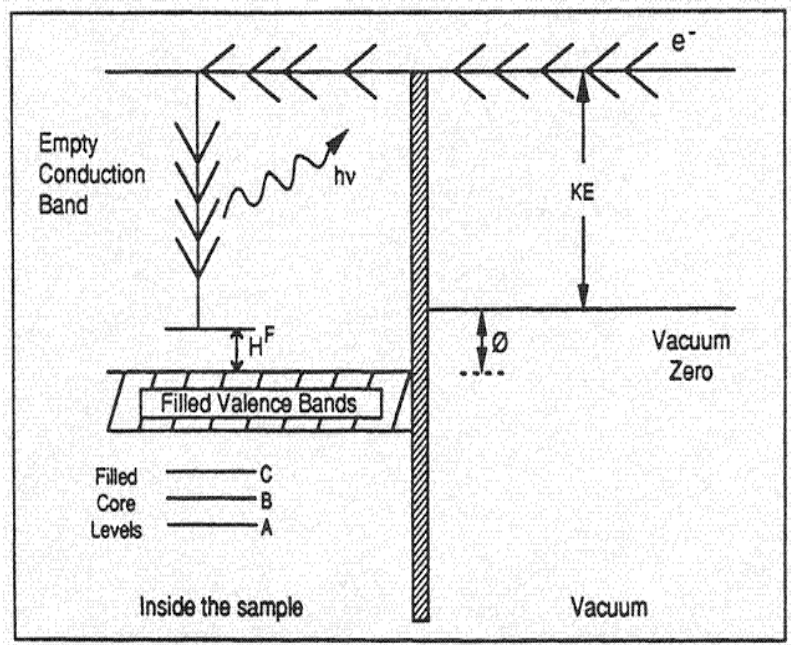

Figure 6

A schematic illustration of the inverse photoemission process is shown here. 


\section{IIIb. Fano Effect Measurements}

The acid test of the "magnetic polarization" model of Pu electronic structure will be the spin dependence. Using the Fano Effect (Double Polarization Photoelectron Dichroism), we may see a strong spin dependence in Non-Magnetic Pu.

The Fano Effect is the emission of spin polarized electrons by NONMAGNETIC materials, when excited by circularly polarized photons, as predicted by U. Fano [15] and measured by U. Heinzmann, J. Kessler, and J. Lorenz [16]. Fano Dichroism PES is the ideal technique with which to probe for such a dynamically shielded moment, with (1) a probe time on the scale of $10^{-18}$ seconds and (2) the capability to see spin effects in nonmagnetic materials!

We are confident that we can see a Fano Effect in non-mag polycrystalline $\mathrm{Pu}$, because we have already observed preliminary evidence of one in non-mag polycrystalline Ce. (See Figure 7.) The spectra in Figure 7 are essentially the fingerprint of a Kondo system. Thus, if $\mathrm{Pu}$ has no spin and orbital polarization but is "merely" Kondo shielded, we should observe this type of spectrum for $\mathrm{Pu}$. Either way, we get the answer.

Plan to resolve the $\mathrm{Pu} 5 \mathrm{f}$ question

- We will be performing the linear dichroic variant of the Fano Effect in-house, using a new hyper- intense He lamp and true spin detection. (Figure 8)

- We will start with polycrystalline Ce films and then move on to polycrystalline $\mathrm{Pu}$.

- By operating at the He II energy $(40.8 \mathrm{eV})$, we will be sitting on a maximum in the $\mathrm{Pu} 5 \mathrm{f}$ cross section.

- By working at LLNL, we can tap into the Pu sample handing and safety expertise of CMS/LLNL, i.e., Mark Wall. 

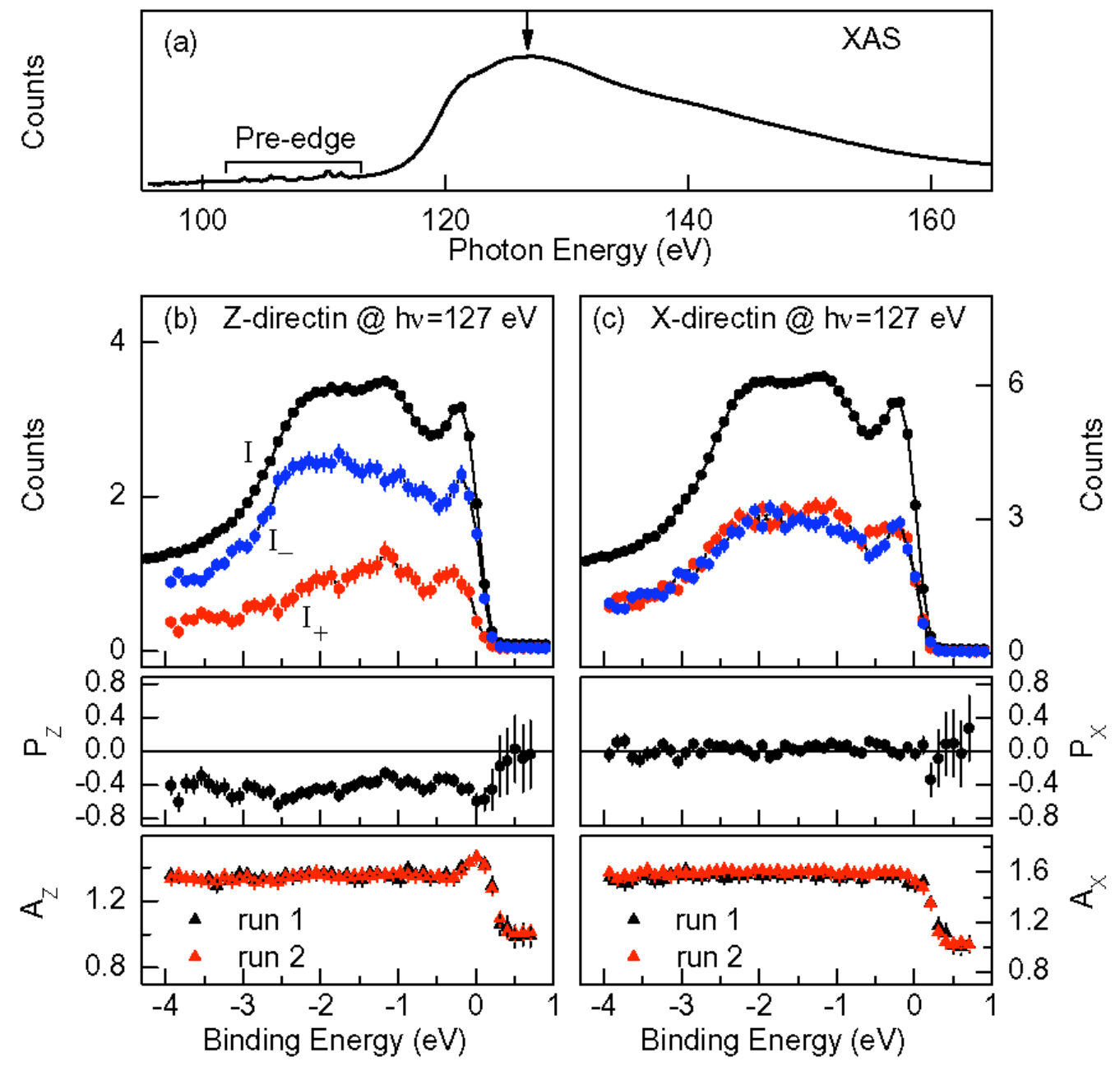

Figure 7

Fano Effect measurements of Ce are exhibited here. Panel a shows the $4 \mathrm{~d}$ to $4 \mathrm{f} x$-ray absorption spectroscopy (XAS), with an arrow indicating the location in energy space where the spin resolved photoelectron spectroscopy (PES) was performed. Panel b contains the spin resolved spectra $\left(\mathrm{I}_{-}, \mathrm{I}_{+}\right)$, spin integrated spectrum (I), spin polarization $\left(\mathrm{P}_{\mathrm{Z}}\right)$ and spin asymmetry $\left(\mathrm{A}_{\mathrm{Z}}\right)$ along the $\mathrm{z}$-direction, which is the direction of propagation of the photon and parallel or anti-parallel to the helicity of the photons. Panel $\mathrm{c}$ has the corresponding quantities for the $\mathrm{x}$ direction. A zero spin effect is expected and observed along the $\mathrm{x}$ direction. For more detail, please see References 5 and 17. 


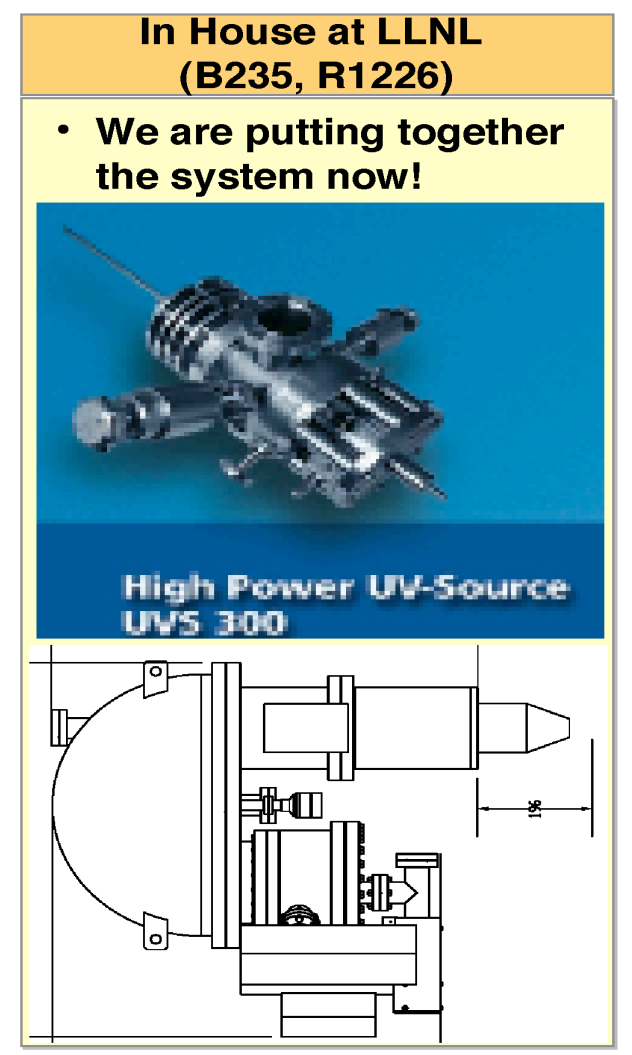

\section{Figure 8}

Instrumentation for the in-house Pu Fano Measurements.

\section{Acknowledgements}

This work was performed under the auspices of the U.S. DOE by Univ. of California, Lawrence Livermore National Laboratory under contract W-7405-Eng-48.

\section{References}

1. J.G. Tobin, B.W. Chung, R. K. Schulze, J. Terry, J. D. Farr, D. K. Shuh, K. Heinzelman, E. Rotenberg, G.D. Waddill, and G. Van der Laan, "Resonant Photoemission in f-electron Systems: Pu and Gd", Phys. Rev. B 68, 1155109 (October 2003).

2. K.T. Moore, M.A. Wall, A.J. Schwartz, B.W. Chung, D.K. Shuh, R.K. Schulze, and J.G. Tobin, "The Failure of Russell-Saunders Coupling in the $5 \mathrm{f}$ States of Plutonium", Phys. Rev. Lett. 90, 196404 (May 2003).

3. G. van der Laan, K.T. Moore, J.G. Tobin, B.W. Chung, M.A. Wall, and A.J. Schwartz, ,Applicability of the spin-orbit sum rule for the actinide $5 \mathrm{f}$ states," Phys. Rev. Lett. 93, 097401 (Aug 2004).

4. J.G. Tobin, K.T. Moore, B.W. Chung, M.A. Wall, A.J. Schwartz, G. van der Laan, and A.L. Kutepov, "Competition Between Delocalization and Spin-Orbit Splitting in the Actinide $5 f$ States," Phys. Rev. B 72, 085109 (2005). 
5. J.G. Tobin, S.A. Morton, B.W. Chung, S.W. Yuand G.D. Waddill, "Spin-Resolved Electronic Structure Studies of Non-Magnetic Systems: Possible Observation of the Fano Effect in Polycrystal Ce," submitted to Physica B, Proceedings of SCES05, Vienna, Austria, July 2005.

6. J.G. Tobin, M.T. Butterfield, N.E. Teslich Jr., R.A. Bliss, M.A. Wall, A.K. McMahan, B.W. Chung, A.J. Schwartz, "Using Nano-focussed Bremstrahlung Isochromat Spectroscopy (nBIS) to Determine the Unoccupied Electronic Structure of Pu," submitted to the Royal Society of Chemistry, Proceedings of the Actinides 2005 Meeting, Manchester, UK, July 2005.

7. B.W. Chung, A.J. Schwartz, B.B. Ebbinghaus, M.J. Fluss, J.J. Haslam, K.J.M. Blobaum, and J.G. Tobin, "Spectroscopic Signature of Aging in $\mathrm{F} \mathrm{Pu}(\mathrm{Ga})$," submitted to Europhysics Letters; B.W. Chung et al, MRS Symp. Proc. 2006.

8. P. Soderlind and B. Sadigh, Phys. Rev. Lett. 92, 185702 (2004).

9. P. Soderlind, A. Landa, and B. Sadigh, Phys Rev. B 66, 205109 (2002).

10. B. Sadigh, P. Soderlind and W. Wolfer, Phys. Rev. B 68, 241101R (2003).

11. G. Kotliar and D. Vollhardt, Physics Today 57, 532004.

12. S.Y. Saravsov, G. Kotliar and E. Abrahams, Nature 410, 793 (2001).

13. Y. Baer and J.K. Lang, Phys. Rev B 21, 2060 (1980).

14. M. Penicaud, J. Phys. Condensed Matter 9, 6341 (1990).

15. U. Fano, Phys. Rev. 178, 131 (1969); 184, 250 (1969).

16. U. Heinzmann, J. Kessler, and J. Lorenz, Phys. Rev. Lett. 25, 1325 (1970).

17. S.W. Yu, T. Komesu, B.W. Chung, G.D. Waddill, S.A. Morton, and J.G. Tobin, "Study of the felectron correlations in nonmagnetic Ce by means of spin resolved resonant photoemission," to be submitted to Phys. Rev. B, 2005. 